\title{
A NEW PROOF FOR THE LÉVY CONSTRUCTION OF SECOND KIND FOR STABLE LAWS
}

\section{Neuenschwander ${ }^{1}$}

We give a direct proof for the "Lévy construction of second kind" for stable laws on the real line without referring to the construction of "first kind."

\section{Introduction}

Let $X$ be a real-valued non-gaussian $\alpha$-stable random variable. It is well known that this is the case iff the Fourier transform (characteristic function) of $X$ has the form

$$
\begin{aligned}
\varphi_{X}(u)= & \exp \left(i u \gamma+c_{-} \int_{-\infty}^{0}\left(e^{i u x}-1-\frac{i u x}{1+x^{2}}\right)|x|^{-(1+\alpha)} d x+\right. \\
& \left.+c_{+} \int_{0}^{\infty}\left(e^{i u x}-1-\frac{i u x}{1+x^{2}}\right) x^{-(1+\alpha)} d x\right), \quad u \in \mathbb{R},
\end{aligned}
$$

with $0<\alpha<2, \gamma \in \mathbb{R}, c_{-}, c_{+} \geqslant 0, c_{-}+c_{+}>0$.

Possible "constructions" of $X$ are the so-called Lévy constructions of "first" and "second kind." These are the following.

Assume $0<\alpha<2$. Let $\left\{N_{t}\right\}_{t \geqslant 0}$ be a Poisson process with parameter $\lambda>0$ and suppose $\Gamma_{j}$ is the time of the $j$ th jump of $\left\{N_{t}\right\}_{t \geqslant 0}$. Suppose $\left\{Y_{j}\right\}_{j \in \mathbb{N}}$ is a sequence of i.i.d. $\{-1,1\}$-valued random variables that is independent of the process $\left\{N_{t}\right\}_{t \geqslant 0}$ and such that $P\left(Y_{j}=1\right)=p$. Put $a_{j}:=0$ for $j \leqslant 1 / \alpha$ and $a_{j}:=E\left(Y_{j}\right) E\left(\Gamma_{j}^{-1 / \alpha}\right)$ for $j>1 / \alpha$. Set

$$
S_{n}(\alpha, \lambda, p, \gamma):=\gamma+\sum_{j=1}^{n}\left(\Gamma_{j}^{-1 / \alpha} Y_{j}-a_{j}\right) .
$$

Theorem 1 (Lévy construction of second kind). The sum $S_{n}(\alpha, \lambda, p, \gamma)$ converges to some $S(\alpha, \lambda, p, \gamma)$ a.s. as $n \rightarrow \infty$, and $S(\alpha, \lambda, p, \gamma)$ exhausts all (nondegenerate) $\alpha$-stable laws as $(\lambda, p, \gamma) \in] 0, \infty[\times[0,1] \times \mathbb{R}$.

For the "Lévy construction of first kind," one just uses that

$$
\mathcal{L}\left(\left(\Gamma_{1}, \Gamma_{2}, \ldots, \Gamma_{n}\right) \mid \Gamma_{n+1}=t\right)=\mathcal{L}\left(\left(U_{[n: 1]}, U_{[n: 2]}, \ldots, U_{[n: n]}\right)\right),
$$

where $U_{[n: 1]}<U_{[n: 2]}<\ldots<U_{[n: n]}$ denotes the increasing order statistics of independent random variables $U_{1}, U_{2}, \ldots, U_{n}$ distributed uniformly on $[0, t]$. Write

$$
F_{t}(\alpha, \lambda, p, \gamma):=\gamma+\sum_{j=1}^{N_{t}}\left(U_{\left[N_{t}: j\right]}^{-1 / \alpha} Y_{j}-a_{j}\right) .
$$

Then the "Lévy construction of first kind" is the following:

Theorem 2 (Lévy construction of first kind). The sum $F_{t}(\alpha, \lambda, p, \gamma)$ converges weakly to some $F(\alpha, \lambda, p, \gamma)$ as $t \rightarrow \infty$, and $F(\alpha, \lambda, p, \gamma)$ exhausts all (nondegenerate) $\alpha$-stable laws as $(\lambda, p, \gamma) \in] 0, \infty[\times[0,1] \times \mathbb{R}$.

The classical proof of Theorem 1 proceeds in the manner that one first verifies Theorem 2 by calculating the Fourier transform of $F_{t}(\alpha, \lambda, p, \gamma)$, then uses (1), and at the end takes the limit as $t \rightarrow \infty$. In other words, the Lévy construction of second kind is deduced from that of the first kind by the equivalence (1). From the pedagogical

\footnotetext{
${ }^{1}$ Université de Lausanne, Ecole des Hautes Etudes Commerciales, Institut de Sciences Actuarielles, CH-1015 Lausanne, and Universität Bern, Insitut für mathematische Statistik und Versicherungslehre, CH-3012 Bern, Switzerland. E-mail: daniel.neuenschwander@bluewin.ch
}

Proceedings of the XXVI International Seminar on Stability Problems for Stochastic Models, Sovata-Bai, Romania, August 27 - September 2, 2006. 
point of vue, this approach has one disadvantage: Although (1) seems to be quite intuitive, a formally absolute correct proof is quite cumbersome to write down. Often, textbooks just give a "proof" using manipulations with differentials (as, e.g., [1]). That is why in this note, we would like to show how a direct approach to the Lévy construction of second kind is possible without using (1). See, e.g., [3-7] for further information and generalizations of the Lévy construction.

\section{Alternative proof of Theorem 1}

The convergence result $S_{n}(\alpha, \lambda, p, \gamma) \stackrel{\text { a.s. }}{\rightarrow} S(\alpha, \lambda, p, \gamma)$ follows from the Three Series Theorem by observing that the sequence $\left\{\Gamma_{j}^{-1 / \alpha}\right\}_{j \geqslant 1}$ behaves as $\{j / \lambda\}_{j} \geqslant 1$ and the conditional variance of $\Gamma_{j}^{-1 / \alpha} Y_{j}$ given $Y_{j}$ is of the form $m \Gamma_{j}^{-2 / \alpha} \sim m_{1} j^{-2 / \alpha}$ as $j \rightarrow \infty$ (cf. [2]). In order to verify the stability of $S(\alpha, \lambda, p, \gamma)$, observe that the addition of $n$ independent copies of $S(\alpha, \lambda, p, \gamma)$ corresponds to a superposition of $n$ independent sequences $\left\{\Gamma_{j}\right\}_{j} \geqslant 1$, i.e., to the addition of $n$ independent copies of the Poisson process $\left\{N_{t}\right\}_{t \geqslant 0}$, which is equivalent to the multiplication of the intensitiy parameter $\lambda$ by $n$. In the sequence of jump times $\left\{\Gamma_{j}\right\}_{j \geqslant 1}$ this corresponds to a division by $n$, hence in the sequence $\left\{\Gamma_{j}^{-1 / \alpha}\right\}_{j \geqslant 1}$ to a multiplication with $n^{1 / \alpha}$. More precisely: Let, for $1 \leqslant k \leqslant n$, processes $\left\{N_{t}^{(k)}\right\}_{t \geqslant 0}$ and $\left\{Y_{j}^{(k)}\right\}_{j \geqslant 1}$ be given as above such that the processes $D^{(k)}:=\left\{\left(N_{t}^{(k)}, Y_{j}^{(k)}\right)\right\}_{t \geqslant 0, j \geqslant 1}$ are i.i.d., $\gamma^{(k)}=\gamma \in \mathbb{R}$, $S^{(k)}(\alpha, \lambda, p, \gamma)$ as above. Then

$$
\begin{aligned}
\mathcal{L}\left(\sum_{k=1}^{n} S^{(k)}(\alpha, \lambda, p, \gamma)\right) & =\mathcal{L}\left(n \gamma+\sum_{k=1}^{n} \sum_{j=1}^{\infty}\left(\left(\Gamma_{j}^{(k)}\right)^{-1 / \alpha} Y_{j}^{(k)}-a_{j}\right)\right)= \\
& =\mathcal{L}\left(\tilde{\gamma}+\sum_{j=1}^{\infty} \tilde{\Gamma}_{j}^{-1 / \alpha}\left(\tilde{Y}_{j}-\tilde{a}_{j}\right)\right)
\end{aligned}
$$

where $\left\{\tilde{\Gamma}_{j}\right\}_{j \geqslant 0}\left(\tilde{\Gamma}_{0}:=0\right)$ is defined as a process with independent increments and

$$
\mathcal{L}\left(\tilde{\Gamma}_{j+1}-\tilde{\Gamma}_{j}\right)=\mathcal{L}\left(\tilde{\Gamma}_{1}\right)=\mathcal{L}\left(\min _{1 \leqslant k \leqslant n} \Gamma_{1}^{(k)}\right)
$$

$\tilde{Y}_{j}, \tilde{a}_{j}$ by analogy as above $\left(\tilde{\Gamma}_{j}\right.$ is the time of the $j$ th jump of the superposition of the processes $\left\{N_{t}^{(k)}\right\}_{t \geqslant 0}$, $k=1,2, \ldots, n$; the property that the increments are i.i.d. follows from the fact that the processes $\left\{N_{t}^{(k)}\right\}_{t \geqslant 0}$ are themselves independent processes with i.i.d. increments). Now

$$
\begin{aligned}
P\left(\min _{1 \leqslant k \leqslant n} \Gamma_{1}^{(k)} \geqslant x\right) & =\prod_{k=1}^{n} P\left(\Gamma_{1}^{(k)} \geqslant x\right)=\prod_{k=1}^{n} e^{-\lambda x}=e^{-n \lambda x}= \\
& =P\left(\Gamma_{1}^{(1)} \geqslant n x\right)=P\left(\Gamma_{1}^{(1)} / n \geqslant x\right),
\end{aligned}
$$

i.e., $\mathcal{L}\left(\min _{1 \leqslant k \leqslant n} \Gamma_{1}^{(k)}\right)=\mathcal{L}\left(\Gamma_{1}^{(1)} / n\right)$, hence $\mathcal{L}\left(\tilde{\Gamma}_{1}^{-1 / \alpha}\right)=\mathcal{L}\left(n^{1 / \alpha}\left(\Gamma_{1}^{(1)}\right)^{-1 / \alpha}\right)$. Thus

$$
\begin{aligned}
\mathcal{L}\left(\sum_{k=1}^{n} S^{(k)}(\alpha, \lambda, p, \gamma)\right) & =\mathcal{L}\left(\tilde{\gamma}+n^{1 / \alpha} S^{(1)}(\alpha, \lambda, p, 0)\right)= \\
& =\mathcal{L}\left(\tilde{\gamma}+n^{1 / \alpha} S(\alpha, \lambda, p, 0)\right) .
\end{aligned}
$$

Since this is true for all $n \geqslant 1$, this means that $S(\alpha, \lambda, p, \gamma)$ obeys an $\alpha$-stable law.

It remains to show that every $\alpha$-stable law is of the form $\mathcal{L}(S(\alpha, \lambda, p, \gamma))$. It holds that

$$
S(\alpha, 1,1,0) \stackrel{a . s .}{=} \lim _{t \rightarrow \infty} S_{t}(\alpha, 1),
$$

where

$$
S_{t}(\alpha, \lambda):=\sum_{j=1}^{N_{t}} \Gamma_{j}^{-1 / \alpha}-\sum_{j=1}^{\lambda t} a_{j} .
$$

Here $\left\{\Gamma_{j}\right\}_{j \geqslant 1}$ as above with parameter $\lambda$. Observe that $N_{t}-\lambda t \stackrel{a . s .}{=} o\left(t^{1 / \alpha}\right), t \rightarrow \infty$, by the Law of the Iterated Logarithm and thus $\sum_{j=1}^{N_{t}} a_{j}-\sum_{j=1}^{\lambda t} a_{j} \stackrel{a . s .}{\rightarrow} 0, t \rightarrow \infty$ (cf. [2]). For every $n \geqslant 1$ we have that

$$
\mathcal{L}\left(S_{t}(\alpha, 1)\right)=\mathcal{L}\left(S_{t}(\alpha, 1 / n)+b_{n}\right)
$$


for suitable $b_{n} \in \mathbb{R}$, i.e. $S_{t}(\alpha, 1)$ is infinitely divisible. Since for all $t \geqslant 0$ the Lévy measure in the LévyHinčin formula of $\mathcal{L}\left(S_{t}(\alpha, 1)\right)$ is concentrated on $[0, \infty[$, the same must hold for the limit $\mathcal{L}(S(\alpha, 1,1,0)$ ) (see e.g. [1, Theorem 9.22]), hence the Fourier transform of $\mu^{(0)}:=\mathcal{L}(S(\alpha, 1,1,0))$ is of the form

$$
\hat{\mu}^{(0)}(u)=\exp \left(i u \gamma^{(0)}+c_{+}^{(0)} \int_{0}^{\infty}\left(e^{i u x}-1-\frac{i u x}{1+x^{2}}\right) x^{-1+\alpha} d x\right)
$$

for some $c_{+}^{(0)}>0$. Now take any (nondegenerate) $\alpha$-stable law $\mu$ given by the Fourier transform

$$
\hat{\mu}(u)=\exp \left(i u \gamma^{(0)}+c_{-} \int_{-\infty}^{0}\left(e^{i u x}-1-\frac{i u x}{1+x^{2}}\right)|x|^{-1+\alpha} d x+c_{+} \int_{0}^{\infty}\left(e^{i u x}-1-\frac{i u x}{1+x^{2}}\right) x^{-1+\alpha} d x\right)
$$

$\left(c_{-}+c_{+}>0\right)$. Then we have

$$
\mu=\mathcal{L}\left(c_{+}^{\prime} S^{\prime}(\alpha, 1,1,0)-c_{-}^{\prime} S^{\prime \prime}(\alpha, 1,1,0)+\gamma^{\prime}\right)
$$

with $c_{+}^{\prime}:=\left(c_{+} / c_{+}^{(0)}\right)^{1 / \alpha}$ and $c_{-}^{\prime}:=\left(c_{-} / c_{+}^{(0)}\right)^{1 / \alpha}$, where $S^{\prime}(\alpha, 1,1,0)$ and $S^{\prime \prime}(\alpha, 1,1,0)$ are i.i.d. random variables obeying the law $\mathcal{L}(S(\alpha, 1,1,0))$. However,

$$
\mathcal{L}\left(c_{+}^{\prime} S^{\prime}(\alpha, 1,1,0)-c_{-}^{\prime} S^{\prime \prime}(\alpha, 1,1,0)+\gamma^{\prime}\right)=\mathcal{L}\left(S\left(\alpha,\left(c_{+}+c_{-}\right) / c_{+}^{(0)}, c_{+} /\left(c_{+}+c_{-}\right), \gamma^{\prime}\right)\right),
$$

i.e., $\mu$ has indeed a Lévy construction of the second kind.

\section{Acknowledgements}

This paper is drawn from a part of the authors diploma work written under the direction of Professor Henri Carnal at the University of Bern in 1987. The author would like to thank Professor Carnal for his guidance and help.

\section{REFERENCES}

1. L. Breiman, Probability. Addison-Wesley, New York (1968).

2. H. Carnal, "Les variables aléatoires de loi stable et leur représentation selon P. Lévy," in: Probability Measures on Groups VIII, H. Heyer (ed.), Springer, Berlin (1986), pp. 24-33.

3. M. Hahn, W. N. Hudson, and J. A. Veeh, "Operator stable laws: Series representations and domains of normal attraction," J. Theor. Probab., 2, No. 1, 3-35 (1989).

4. A. Janssen, "The domain of attraction of stable laws and extreme order statistics," Prob. Math. Stat., 10, No. 2, 205-222 (1989).

5. R. Le Page, M. Woodroofe, and J. Zinn, "Convergence to a stable distribution via order statistics," Ann. Prob., 9, No. 4, 624-632 (1981).

6. D. Neuenschwander, "Lightly trimmed products on simply connected step 2-nilpotent Lie groups," in: Probability Measures on Groups and Related Structures XI, H. Heyer (ed.), World Scientific, Singapore (1995), pp. 244-259.

7. D. Neuenschwander, Probability Measures on the Heisenberg Group: Limit Theorems and Brownian Motion. Lecture Notes in Mathematics, Vol. 1630, Springer, Berlin (1996). 